\title{
Razza, condizioni socioeconomiche e gravità dell'oftalmopatia Basedowiana
}

\author{
Michele Marinò
}

Pubblicato online: 2 luglio 2014

(c) Springer International Publishing AG 2014

\section{Commento a:}

Are ethnicity, social grade, and social deprivation associated with severity of thyroid-associated ophthalmopathy?

M.R. Edmunds, J.A. Huntbach, O.M. Durrani.

Ophthal Plast Reconstr Surg (2014) [Epub ahead of print]

In questo studio condotto nel Regno Unito veniva valutata la possibile associazione tra gravità dell'oftalmopatia Basedowiana e la razza e le condizioni socioeconomiche. Esiste infatti un'ipotesi patogenetica sulle malattie autoimmuni, denominata hygiene hypothesis of autoimmunity, peraltro mai dimostrata con certezza, in base alla quale le malattie autoimmuni stesse sarebbero in qualche modo facilitate dalle buone condizioni igieniche.

In questo studio venivano valutati, in tutti i casi di oftalmopatia Basedowiana osservati in un periodo di 14 anni al Birmingham and Midland Eye Centre, la razza e le condizioni socioeconomiche, queste ultime arbitrariamente dedotte dalla zona di residenza, ovvero dal Codice Postale. Tale deduzione è a nostro avviso quanto meno approssimativa, lasciando perdere le implicazioni politiche di una simile scelta, che potrebbe molto facilmente essere considerata scorretta.
La gravità dell'oftalmopatia Basedowiana veniva stabilita sulla base dei criteri dello European Group on Graves' Orbitopathy (EUGOGO). L'oftalmopatia Basedowiana veniva definita di grado medio-grave in tutti i casi che necessitavano di terapia steroidea per via orale, endovenosa o che necessitavano di radioterapia orbitaria o che presentavano neuropatia ottica. In realtà, questi non sono esattamente i criteri applicati da EUGOGO, tenuto conto del fatto che la terapia con glucocorticoidi per via orale a basse dosi viene comunemente somministrata ai pazienti con oftalmopatia Basedowiana di grado lieve sottoposti alla terapia con radioiodio. È ovvio che questo bias sia sufficiente per condizionare in modo decisivo i risultati dello studio.

$\mathrm{Ne}$ consegue che l'interpretazione dei risultati stessi, che sembrano mostrare un'associazione significativa tra oftalmopatia Basedowiana e standard socioeconomici bassi (peraltro, al contrario di quanto postulato dalla hygiene hypothesis of autoimmunity) è quanto meno difficile e francamente chi scrive trova impossibile giungere ad alcuna conclusione. L'unica possibile spiegazione, se questi risultati sono attendibili, è che le persone con standard socioeconomici bassi abbiano accesso alle procedure diagnostiche e alle cure più tardivamente $\mathrm{e}$, per questo motivo, presentino un quadro clinico più grave. 